\title{
Surgical Resection of Amygdala and Uncus
}

\author{
Naotaka UsUI, ${ }^{1}$ Akihiko KONDO, ${ }^{1}$ Naoki NITTA, ${ }^{1}$ \\ Takayasu TOTTORI, ${ }^{1}$ and Yushi INOUE ${ }^{1}$
}

\author{
${ }^{1}$ National Epilepsy Center, Shizuoka Institute of Epilepsy and Neurological Disorders, \\ Shizuoka, Shizuoka, Japan
}

\begin{abstract}
The amygdala and uncus are located close to important neurovascular structures. We describe a safe technique for resection of amygdala and uncus. Under general anesthesia, the patient is positioned supine, with the head rotated approximately 20 degrees to the unoperated side and slightly extended. By using a trans-anterior $T_{1}$ subpial approach, the inferior horn of the lateral ventricle is opened, and hippocampectomy is performed. We treat an imaginary plane formed by the inferior circular sulcus of the insula, the endorhinal sulcus, and the inferior choroidal point as the upper border of amygdalar resection. After confirming the position of the inferior choroidal point, the border between the temporal stem and uncus is exposed from anterior to posterior. This border is continuous with the endorhinal sulcus. By exposing the endorhinal sulcus, the anterior choroidal artery and optic tract can be visualized. The amygdala is disconnected through complete exposure of the endorhinal sulcus to the inferior choroidal point. After the lateral side of the uncus is disconnected, the amygdala and uncus are removed en bloc. Since April 2014, we have used the described procedure to remove amygdalar-uncal lesions in 15 patients. The lesion was completely removed in all cases without complications. Histological specimens were obtained in all cases. Our procedure enables safe and complete removal of amygdalar-uncal lesions. Imagining the plane formed by the inferior circular sulcus, inferior choroidal point, and endorhinal sulcus is essential for complete removal of the lesion and for preserving important structures.
\end{abstract}

Key words: selective amygdalohippocampectomy, amygdala, inferior choroidal point, endorhinal sulcus, inferior circular sulcus

\section{Introduction}

In surgery to treat mesial temporal lobe epilepsy, resection of the amygdala and uncus, in addition to the hippocampus, is important for seizure relief. ${ }^{1,2)}$ In cases of brain tumors located within the amygdala and uncus, complete resection of the lesion is extremely important for the survival of the patients. Because the amygdala and uncus are located in the vicinity of important structures, such as the anterior choroidal artery, the optic tract, the oculomotor nerve, and the cerebral peduncle, the careful technique is necessary to provide safe and adequate resection. However, amygdalar-uncal resection has rarely been described in detail in the literature. ${ }^{3-5)}$

Received May 2, 2018; Accepted June 25, 2018

Copyright $\subset 2018$ by The Japan Neurosurgical Society This work is licensed under a Creative Commons AttributionNonCommercial-NoDerivatives International License.
In our institute, we routinely remove the amygdala and uncus in addition to the hippocampus during surgery for mesial temporal lobe epilepsy, because we believe that the amygdala plays an important role in the generation of clinical seizures., ${ }^{1,2)}$ Here, we describe the procedure that we have developed to safely resect the amygdala and uncus.

\section{Methods}

\section{Patients}

From April 2014 to December 2017, temporal lobe resection was performed in 102 patients (selective amygdalohippocampectomy in 65 and anterior temporal lobectomy in 37) in our institute. Of these 102 patients, 15 patients with magnetic resonance imaging (MRI) lesions mainly located in the amygdala and uncus were included. The patients with mesial temporal lobe epilepsy with hippocampal sclerosis and those with nonlesional MRI were excluded. Among the 15 patients, 14 of them (patients 1-14) were suffering from intractable epileptic seizures (Table 1). 
Table 1 Demographics, surgery, and outcomes of the patients

\begin{tabular}{ccccccc}
\hline No & $\begin{array}{c}\text { Age at epilepsy } \\
\text { onset (years) }\end{array}$ & $\begin{array}{c}\text { Age at surgery } \\
\text { (years) }\end{array}$ & Surgery & Pathology & $\begin{array}{c}\text { Follow-up } \\
\text { (years) }\end{array}$ & Seizure outcome \\
\hline 1 & 0 & 5 & ATL & DNT & 3 & Ia \\
2 & 31 & 37 & AHE & Gliosis & 2 & IIb \\
3 & 25 & 39 & AHE & AE & 2 & IIb \\
4 & 26 & 39 & AHE & AE & 2 & Ia \\
5 & 30 & 52 & ATL & AE & 2 & Ia \\
6 & 28 & 42 & ATL & AE & 1 & Seizure-free \\
7 & 22 & 24 & AHE & AE & 1 & Worthwhile improvement \\
8 & 9 & 42 & AHE & AE & 1 & No worthwhile improvement \\
9 & 19 & 24 & ATL & GG & 1 & Seizure-free \\
10 & 19 & 37 & ATL & AE & 1 & Rare seizure \\
11 & 22 & 26 & AHE & GG & 1 & Seizure-free \\
12 & 5 & 14 & ATL & DNT & $<1$ & NA \\
13 & 16 & 28 & AHE & AE & $<1$ & NA \\
14 & 0 & 23 & ATL & GG & $<1$ & NA \\
15 & NA & 14 & ATL & Low grade glioma & 1 & NA \\
\hline
\end{tabular}

AE: amygdalar enlargement, AHE: selective amygdalohippocampectomy, ATL: anterior temporal lobectomy, DNT: dysembryoplastic neuroepithelial tumor, GG: ganglioglioma, NA: not applicable.

Craniotomy, trans-anterior $\mathrm{T}_{1}$ subpial approach, and opening the inferior horn

Under general anesthesia, the patient is positioned supine, with the head rotated approximately 20 degrees toward the unoperated side, slightly extended, and secured with a Mayfield clamp. A question mark-shaped skin incision is made, starting just above the zygoma and about $10 \mathrm{~mm}$ anterior to the tragus for exposure of the temporofrontal area (Fig. 1, left). Sufficient exposure of the pterion is important. A temporal craniotomy is performed (Fig. 1, right), and the sphenoid ridge is removed with a rongeur to the level of the frontal base. The dura is opened in a semicircular fashion above the Sylvian fissure. Exposure of the anterior part of the superior temporal gyrus $\left(\mathrm{T}_{1}\right)$ is important.

We adopt a trans-anterior $\mathrm{T}_{1}$ subpial approach for selective amygdalohippocampectomy. The anterior part of $\mathrm{T}_{1}$ is dissected subpially from the pia of the Sylvian fissure to expose the anterior portion of the temporal stem, the M2 segment of the middle cerebral artery (MCA), and the inferior circular sulcus of the insula. An incision of approximately $20 \mathrm{~mm}$ along the anteroposterior axis of $\mathrm{T}_{1}$ between the two temporal arteries (temporopolar artery and anterior temporal artery) is enough for the subsequent procedures (Fig. 2, left). A vein can frequently be seen at the inferior circular sulcus. The middle cerebral artery should be protected with oxycel cotton and a large cotton pad. Throughout the procedure, care should be taken to avoid compressing the MCA.

The inferior horn of the lateral ventricle is approached by aspirating the most anterior part of the temporal stem. The entry point is made approximately $5 \mathrm{~mm}$ lateral to the inferior circular sulcus, and 5-10 $\mathrm{mm}$ posterior from the tip of the temporal stem (Fig. 2, left). The angle of depression of the microscope is approximately 45 degrees. Use of neuronavigation is helpful for this step. Usually, the inferior horn is opened approximately $10 \mathrm{~mm}$ in depth. If grey matter is encountered, the grey matter corresponds to the amygdala or the temporal basal cortices, and the direction of the approach should be adjusted. Once the ventricle is entered, the hippocampus is identified, and the ventricular opening is enlarged anteriorly. Several ependymal veins are coagulated and divided. The amygdala is identified as the superomedial wall of the inferior horn, which bulges into the ventricle. At this step, we do not remove the amygdala. Posteriorly, it is sufficient to open the ventricle until the posterior edge of the bulge of the amygdala can easily be visualized. A more posterior opening of the inferior horn may lead to contralateral upper quadrantoanopsia, although the patient may not be aware of it.

\section{Removal of the hippocampus}

Just medial to the posterior edge of the bulge of the amygdala into the ventricle, the choroid plexus 

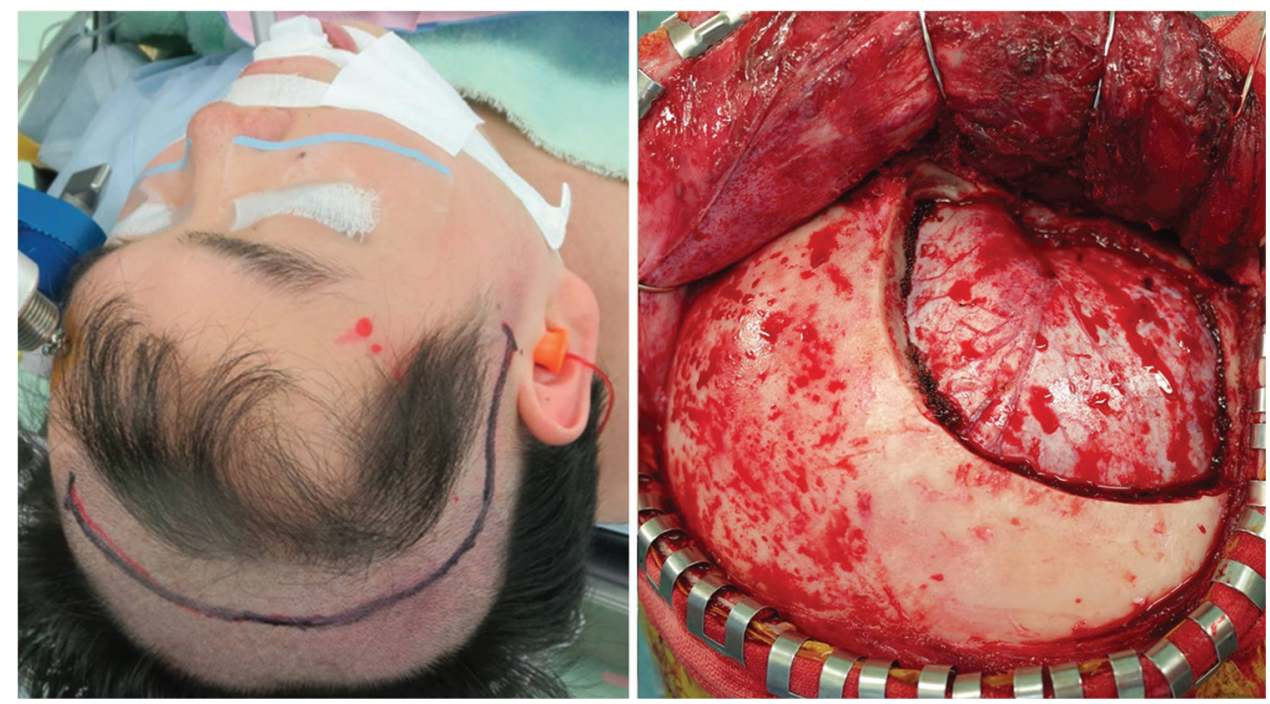

Fig. 1 Scalp incision and craniotomy (right-sided operation). Left: Scalp incision. Right: Craniotomy.
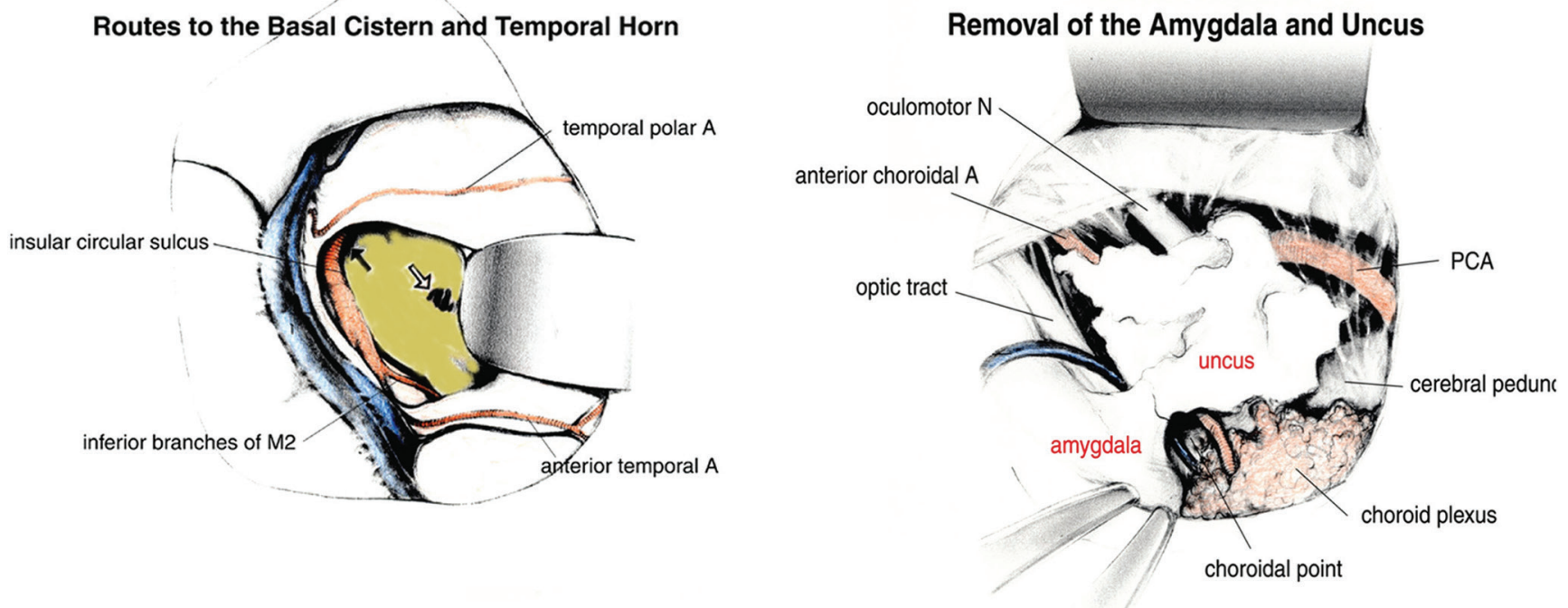

Fig. 2 Schema showing trans-anterior $T_{1}$ subpial approach, and removal of the amygdala and uncus (right-sided operation) (modified from Mihara T, Noushinkeigeka Shujutu Atlas, Igaku Shoin, 2005). Left: A black arrow indicates the route to the cistern, and a white arrow indicates the entry point to the inferior horn. Right: The inferior choroidal point is the superior-posterior limit of the amygdala. The vein draining into the basal vein is colored blue. The vein is a good landmark for the anterior border of the amygdala.

is gently retracted medially with a small cottonoid, and the hippocampus is gradually retracted laterally by using a self-retaining retractor to visualize the flattest and widest part of the fimbria, which is avascular. The radial distribution of the veins running over the surface of the hippocampus is also helpful for identifying the fimbria. The coagulating power of bipolar forceps is lowered slightly. By coagulation and gentle aspiration of the fimbria, the hippocampal sulcus is exposed, and the medial side of the parahippocampal gyrus can be seen. By following the medial edge of the parahippocampal gyrus, the uncal sulcus, which separates the uncus and parahippocampal gyrus anteriorly, is exposed. The anterior part of the hippocampus is cut diagonally until the end of the uncal sulcus is reached. Where the uncal sulcus disappears, the anterior part of the parahippocampal gyrus, namely the entorhinal area, is aspirated, disconnecting it from the uncus, which is located medially. The edge of the tentorium cerebelli is identified. The arteries and veins in the hippocampal sulcus are coagulated and divided. The medial part of the parahippocampal gyrus is aspirated subpially from the pia of the 
ambient cistern until the inferolateral trunk of the P2 segment of the posterior cerebral artery is identified. The inferolateral trunk is a good landmark for the posterior limit of the hippocampal resection.

Then, on the lateral side of hippocampus, the collateral sulcus is exposed by aspirating the collateral eminence. After the temporal base is reached, the posterior transection of the hippocampus is carried out at the level of inferolateral trunk of the posterior cerebral artery. The posterior end of the hippocampal resection approximately corresponds to the level of the lateral mesencephalic sulcus of the midbrain on the anteroposterior axis. After posterior transection of the hippocampus, by following the unnamed sulcus between the hippocampus and collateral eminence, the lateral side of the hippocampus is disconnected. Finally, the hippocampus is removed en bloc.

\section{Removal of the amygdala and the uncus}

After removal of the hippocampus, the inferior choroidal point (Fig. 3, left) is identified inside the inferior horn. Gentle retraction of the choroid plexus posteriorly and the amygdala anteriorly by using a small cottonoid will facilitate visualization (Fig. 3, left). In addition, the operating table can be slightly rotated to the contralateral side. We consider the inferior choroidal point to be the superior-posterior limit of the amygdala (Fig. 2, right). Its location should be kept in mind during the following procedures, and care should be taken not to disturb the areas posterior and superior to this point. After locating the inferior choroidal point, the border between the anterior part of the temporal stem and the uncus has to be exposed carefully from anterior to posterior. This border is connected to the endorhinal sulcus. We consider the plane formed by the inferior circular sulcus of the insula, the inferior choroidal point, and the endorhinal sulcus to be the upper border for the amygdalar resection (Fig. 2, right, Fig. 4). The temporal stem has to be aspirated carefully to make the plane. During the procedure, the vein draining into the basal vein is often seen (Fig. 2, right). The vein is a good landmark for the anterior border of the amygdala. The anterior choroidal artery and the optic tract can be visualized through the pia and arachnoid. The pia and arachnoid should be preserved as much as possible for protecting neurovascular structures. Careful exposure of the endorhinal sulcus continues posteriorly until it reaches the inferior choroidal point.

After disconnecting the lateral side of the uncus along the free edge of the tentorium cerebelli, the uncus is dissected subpially from both the anterior and posterior sides. During the subpial dissection of the anterior side of the uncus, the oculomotor nerve can be identified. Finally, the amygdala and uncus are removed en bloc. After resection of the amygdala and uncus, the anterior choroidal artery, posterior cerebral artery, optic tract, oculomotor nerve, and cerebral peduncle can be identified through the pia and arachnoid (Fig. 3, right).

Following careful establishment of hemostasis, the dura is closed and the bone flap replaced with fixation devices. The muscle and skin are closed layer by layer in the usual fashion.

\section{Results}

In our institute, 15 patients with amygdalar-uncal lesions have undergone resection from April 2014 to December 2017 (Table 1). Age at surgery ranged from 5 to 52 years (mean 29.7 years). Selective amygdalohippocampectomy was performed in seven
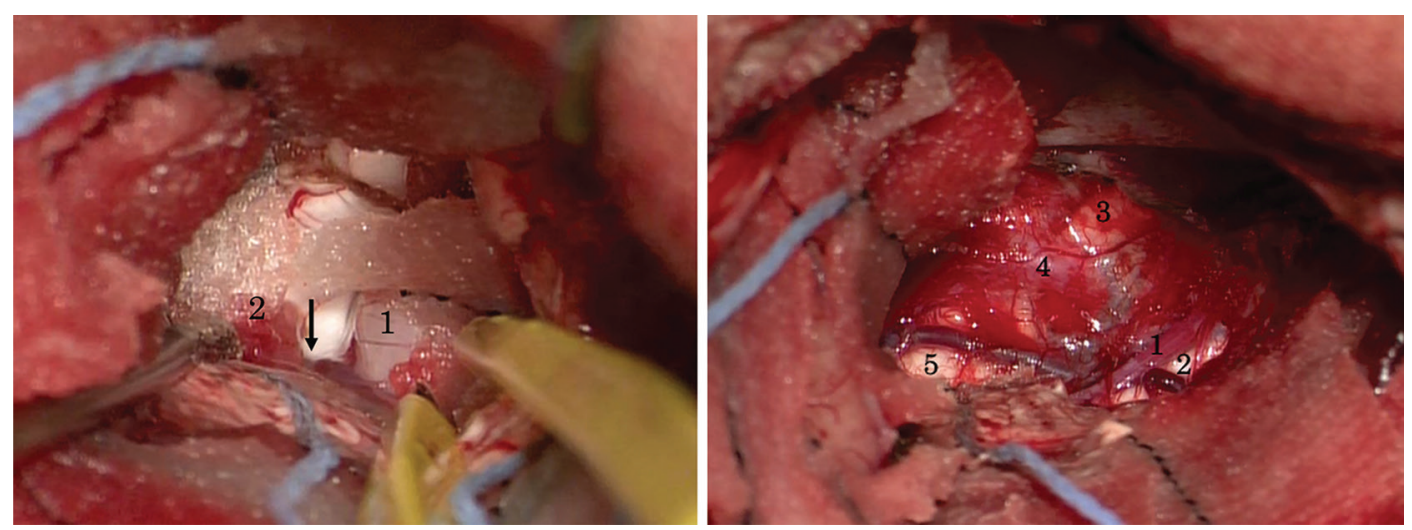

Fig. 3 Intraoperative identification of the inferior choroidal point, and intraoperative view after amygdalaruncal resection (left-sided operation). Left: The amygdala (1) is retracted anteriorly, and the choroid plexus (2) is retracted posteriorly to reveal the inferior choroidal point (arrow). Right: The anterior choroidal artery (1), optic tract (2), oculomotor nerve (3), posterior cerebral artery (4), and cerebral peduncle (5) are identified. 
patients, and anterior temporal lobectomy was performed in the remaining eight patients. In these eight patients, anterior temporal lobectomy was performed because the structural lesion, putative epileptogenic zone, or both extended beyond the mesial temporal structures. In all patients, the amygdalar-uncal lesions were completely removed. No postoperative complications were observed. The surgical specimens of the amygdalar-uncal lesions were sufficient for histology.

\section{Case presentation}

A 37-year-old female with an unremarkable family history experienced head trauma in a traffic accident when she was 15 years old, with transient loss of consciousness. Head MRI taken at the time of the accident incidentally revealed a high-intensity area in the left mesial temporal region on $\mathrm{T}_{2}$-weighted images. She started to have secondarily generalized seizures during sleep at the age of 31 years. The seizures occurred approximately once per month and were intractable to medication, including valproate and carbamazepine. Video-EEG monitoring revealed that her seizures were arising from the left temporal region. FLAIR MRI revealed a high-intensity lesion in the left mesial temporal lobe. The lesion was mainly located in the left amygdalar-uncal region (Fig. 4, upper row). Left selective amygdalohippocampectomy, including the lesion, was performed without complication. Postoperative MRI showed complete removal of the lesion (Fig. 4, lower row). Histopathology of the resected amygdala revealed amygdalar sclerosis characterized by marked glial proliferation and neuron loss. No malignancy was shown. She experienced seizures only rarely after the surgery.

\section{Discussion}

Operative procedures for hippocampectomy have been well described previously. ${ }^{6-12)}$ However, amygdalar-uncal resection has rarely been described in detail in the literature. We therefore, developed our own procedure, which we described here.

The importance of amygdalar resection in mesial temporal lobe epilepsy surgery is controversial. Although Goldring et al. found no significant difference in seizure outcome between patients who underwent anterior temporal lobectomy including the amygdala and those in whom the amygdala was not resected ${ }^{13)}$ the importance of the amygdala in clinical expression of temporal lobe seizures has been demonstrated electrophysiologically., ${ }^{1,2)}$
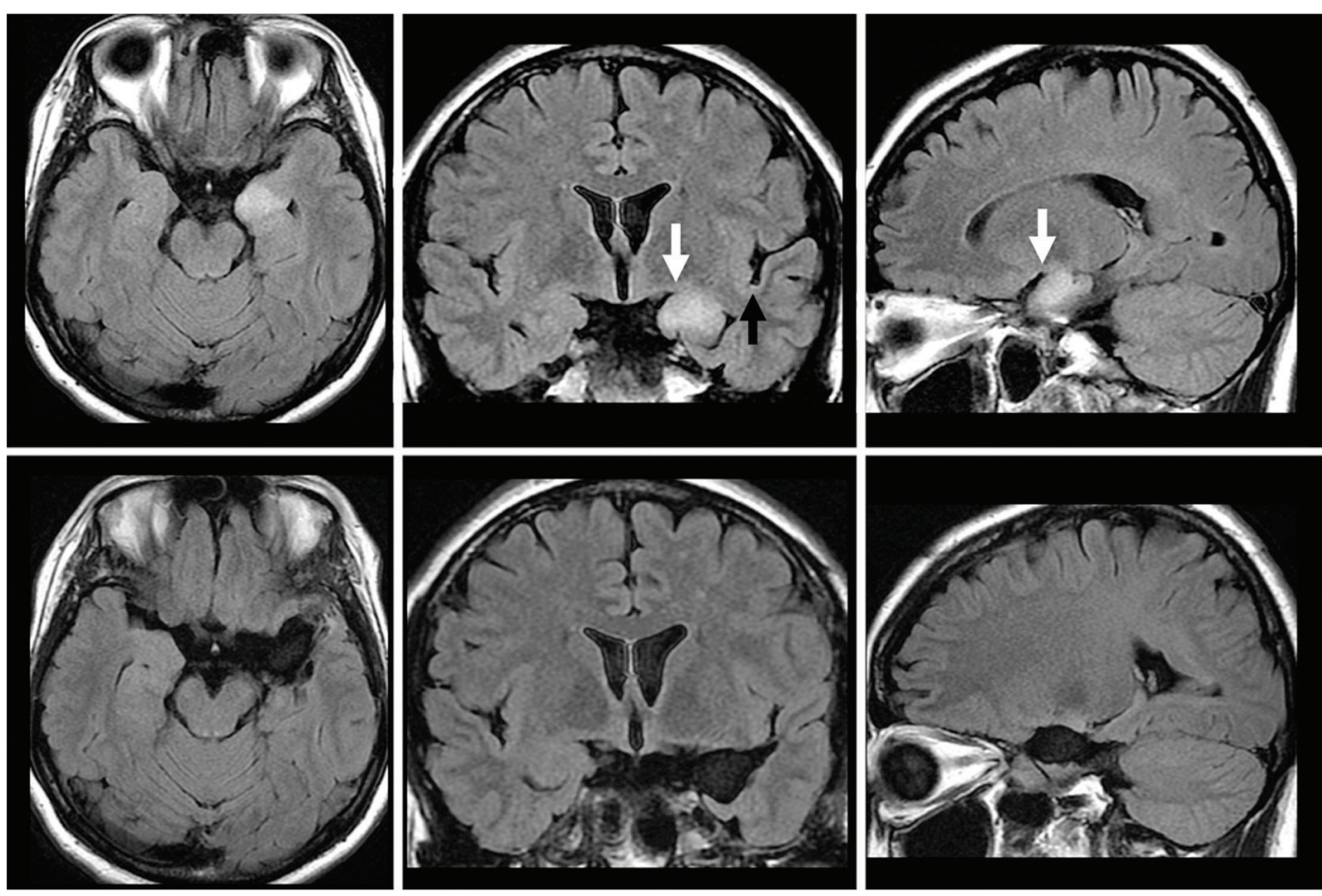

Fig. 4 Pre- and postoperative MRI of the patient. Upper: Preoperative FLAIR MRI of the patient shows a highintensity lesion in the left mesial temporal region, mainly located in the amygdala. A white arrow indicates the endorhinal sulcus, and a black arrow indicates the inferior circular sulcus. Lower: Postoperative FLAIR MRI reveals complete resection of the lesion by left selective amygdalohippocampectomy. The upper border of amygdalar resection corresponds to the line connecting the inferior circular sulcus of the insula and the endorhinal sulcus. 
Amygdalar-uncal resection is difficult because the borders, especially the upper border of the amygdala, are difficult to define. The borders cannot be defined only from the part of the amygdala that is seen inside the ventricle. Tubbs et al. defined the line connecting the MCA point and the anterior choroidal artery (AChoA) point as a guide for amygdalar resection. $\left.{ }^{4}\right)$ They defined the MCA point as the bifurcation of the MCA, and the AChoA point as the inferior choroidal point. The plane defined by the MCA-AChoA line roughly corresponds to our imaginary plane, which is formed by the endorhinal sulcus and inferior circular sulcus and serves as the upper limit of amygdalar resection. Comair used a line joining the endorhinal sulcus and the tip of the choroid plexus as the superior limit of amygdalar resection. $\left.{ }^{5}\right)$ Usually, the vein draining into the basal vein is a good landmark for the anterior margin of the amygdala.

The amygdala is subdivided into a number of nuclei and subnuclei. The cortical and medial nuclei are olfactory centers, whereas the basal, lateral, and central nuclei have limbic functions. ${ }^{14)}$ Completely removing all of the nuclei and subnuclei of the amygdala is impossible. The amygdala is continuous with claustrum and basal ganglia at its superior extent without a clear anatomical border. ${ }^{3)}$ The basal and lateral nuclei are completely resected; however, the most medial part of the amygdala adjoining the striatum cannot be removed. Complete resection is also not possible when the lesion extends superior and medial to the amygdala, but the lesion can be resected safely up to the superior plane we defined here.

Several approaches have been reported for resection of mesial temporal structures, including transsylvian, ${ }^{6)}$ transcortical (trans- $\mathrm{T}_{1}{ }^{7)},-\mathrm{T}_{2}{ }^{8}$ ), transinferior temporal sulcul, ${ }^{9)}$ and subtemporal. ${ }^{10,11)}$ Each approach has some advantages and disadvantages. Several authors reported subtemporal selective amygdalohippocampectomy. ${ }^{10,11)}$ In terms of "selective" resection of mesial temporal structures, the subtemporal approach is the most reasonable. The temporal stem can be preserved in this approach. However, amygdalar-uncal resection may be more difficult in terms of the direction of the approach. Park et al. used an ultrasonic aspirator and did not attempt radical resection of the amygdala in their subtemporal approach. ${ }^{11)}$ En bloc resection of the amygdala may be difficult by this approach. Vajkoczy reported a transsylvian-transcisternal route, whereby the parahippocampal gyrus is directly exposed from its medial aspect, allowing en bloc resection of the mesial temporal structures (amygdala, anterior hippocampus, parahippocampal gyrus, and subiculum). ${ }^{12)}$ However, their approach requires the careful dissection of the oculomotor nerve throughout its entire cisternal course, and they reported that $9 \%$ of patients developed temporary partial oculomotor nerve palsy.

The direction of the approach in our technique is suitable for amygdalar resection. The most important component in our technique is to accurately imagine the plane formed by the inferior circular sulcus and endorhinal sulcus, because the endorhinal sulcus cannot be seen early during the procedure. Other advantages include the protection of neurovascular structures by subpial resection. Unlike many approaches to resecting the amygdala and uncus, our procedures do not use an ultrasonic aspirator, which allows the tissues to be removed en bloc and preserved for histological analysis. By adequately imaging the surgical plane in the early stage of amygdalar-uncal resection, our en bloc resection technique may provide clearer anatomical orientation than using an ultrasonic aspirator. The lesion was completely resected in all of our patients, and no surgical morbidity was observed. Therefore, we think that our technique is suitable for the complete and safe removal of amygdalar-uncal lesions. A cortical incision of $\mathrm{T}_{1}$ of only $15-20 \mathrm{~mm}$ typically is sufficient.

\section{Conclusions}

Our procedure using a trans-anterior $\mathrm{T}_{1}$ subpial approach enables safe and complete removal of amygdalar-uncal lesions. Imagining the plane formed by the inferior circular sulcus of the insula, inferior choroidal point, and endorhinal sulcus is essential for defining the upper border of resection.

\section{Conflicts of Interest Disclosure}

Yushi Inoue received research funding from Health, Labour and Welfare Sciences Research Grants. The remaining authors have no conflict of interest to disclose.

\section{References}

1) Wennberg R, Arruda F, Quesney LF, Olivier A: Preeminence of extrahippocampal structures in the generation of mesial temporal seizures: evidence from human depth electrode recordings. Epilepsia 43: 716-726, 2002

2) Gotman J, Levtova V: Amygdala-hippocampus relationships in temporal lobe seizures: a phase-coherence study. Epilepsy Res 25: 51-57, 1996

3) Tan LA, Byrne RW: Anterior temporal sulcus: a reliable intraoperative landmark for accurately delineating the superior limit of amygdala resection during anterior temporal lobectomy. Stereotact Funct Neurosurg 93: 360-365, 2015 
4) Tubbs RS, Miller JH, Cohen-Gadol AA, Spencer DD: Intraoperative anatomic landmarks for resection of the amygdale during medial temporal lobe surgery. Neurosurgery 66: 974-977, 2010

5) Comair YG: The transsylvian functional hemispherectomy: patient selection and results. In Lüders HO, Comair YG (eds): Epilepsy Surgery, ed 2. Philadelphia, Lippincott Williams and Wilkins, 2001, pp. 699-704

6) Yaşargil MG, Teddy PJ, Roth P: Selective amygdalohippocampectomy. Operative anatomy and surgical technique. In Symon L, Brihaye J, Guidetti B et al. (eds): Advances and Technical Standards in Neurosurgery, vol 12. Vienna, Springer-Verlag, 1985, pp. 93-123

7) Olivier A: Commentary: Cortical resections. In Engel J Jr (ed): Surgical Treatment of the Epilepsies. New York, Raven Press, 1987, pp. 405-416

8) Niemeyer P: The transventricular amygdalohippocampectomy in temporal lobe epilepsy. In Baldwin M, Bailey P (eds): Temporal Lobe Epilepsy. Illinois, CC Thomas Springfield, 1958, pp. 461-482

9) Miyagi Y, Shima F, Ishido K, et al.: Inferior temporal sulcus approach for amygdalohippocampectomy guided by a laser beam of stereotactic navigator. Neurosurgery 52: 1117-1124, 2003

10) Hori T, Tabuchi S, Kurosaki M, Kondo S, Takenobu A, Watanabe T: Subtemporal amygdalohippocampectomy for treating medically intractable temporal lobe epilepsy. Neurosurgery 33: 50-57, 1993

11) Park TS, Bourgeois BF, Silbergeld DL, Dodson WE: Subtemporal transparahippocampal amygdaohippocampectomy for surgical treatment of mesial temporal lobe epilepsy. Technical note. J Neurosurg 85: 1172-1176, 1996

12) Vajkoczy P, Krakow K, Stodieck S, Pohlmann-Eden B, Schmiedek P: Modified approach for the selective treatment of temporal lobe epilepsy: transsylviantranscisternal mesial en bloc resection. J Neurosurg 88: 855-862, 1998

13) Goldring S, Edwards I, Harding GW, Bernardo KL: Results of anterior temporal lobectomy that spares the amygdala in patients with complex partial seizures. J Neurosurg 77: 185-193, 1992

14) Aggeleton JP: The amygdala: Neurobiological Aspects of Emotion, Memory and Mental Dysfunction. New York, Wiley-Liss, 1992

Address reprint requests to: Naotaka Usui, MD, PhD, Department of Neurosurgery, National Epilepsy Center, Shizuoka Institute of Epilepsy and Neurological Disorders, 886 Urushiyama, Aoi-ku, Shizuoka, Shizuoka 420-8688, Japan.

e-mail: usui-nsu@umin.ac.jp 